\title{
Enhancement of Electrochemical Performance of Bilirubin Oxidase Modified Gas Diffusion Biocathode by Porphyrin Precursor
}

\author{
Erica Pinchon, Mary Arugula, Kapil Pant, and Sameer Singhal \\ CFD Research Corporation, 701 McMillian Way, Suite D, Huntsville, AL 35806, USA \\ Correspondence should be addressed to Sameer Singhal; sameer.singhal@cfdrc.com
}

Received 16 December 2017; Revised 12 March 2018; Accepted 28 March 2018; Published 3 June 2018

Academic Editor: Ramasamy Karvembu

Copyright (c) 2018 Erica Pinchon et al. This is an open access article distributed under the Creative Commons Attribution License, which permits unrestricted use, distribution, and reproduction in any medium, provided the original work is properly cited.

\begin{abstract}
Recent studies have focused on tailoring the catalytic currents of multicopper oxidase (MCO) enzymes-based biocathodes to enhance oxygen reduction. Biocathodes modified with natural substrates specific for MCO enzymes demonstrated drastic improvement for oxygen reduction. Performance of 1-pyrenebutanoic acid, succinimidyl ester (PBSE), and 2,5-dimethyl-1phenyl-1H-pyrrole-3-carbaldehyde (Di-Carb) oriented bilirubin oxidase (BOx) modified gas diffusion biocathode has been highly improved by incorporating hematin, a porphyrin precursor as electron transfer enhancement moiety. Hematin modified electrodes demonstrated direct electron transfer reaction of $\mathrm{BOx}$ exhibiting larger $\mathrm{O}_{2}$ reduction in current density in phosphate buffer solution $(\mathrm{pH}$ 7.0) without the need of a mediator. A remarkable improvement in the catalytic currents with 2.5 -fold increase compared to non-hematin modified oriented BOx electrodes was achieved. Moreover, a mediatorless and compartmentless glucose/ $\mathrm{O}_{2}$ biofuel cell based on DET-type bioelectrocatalysis via the BOx cathode and the glucose dehydrogenase (GDH) anode demonstrated peak power densities of $1 \mathrm{~mW} / \mathrm{cm}^{2}$ at $\mathrm{pH} 7.0$ with $100 \mathrm{mM}$ glucose $/ 10 \mathrm{mM}$ NAD fuel. The maximum current density of $1.6 \mathrm{~mA} / \mathrm{cm}^{2}$ and the maximum power density of $0.4 \mathrm{~mW} / \mathrm{cm}^{2}$ were achieved at $300 \mathrm{mV}$ with nonmodified BOx cathode, while $3.5 \mathrm{~mA} / \mathrm{cm}^{2}$ and $1.1 \mathrm{~mW} / \mathrm{cm}^{2}$ of current and power density were achieved with hematin modified cathode. The performance improved 2.4 times which attributes to the hematin acting as a natural precursor and activator for BOx activity enhancement.
\end{abstract}

\section{Introduction}

Biofuel cell technology has received much attention as energy harvesting devices for powering portable devices and microscale electronic systems. Enzymes functionalized with nanomaterials such as carbon nanotubes, graphene, and nanoparticles provide extremely powerful platforms for wide range of biofuel cell applications that are capable of operating independently over a prolonged period of time, without the need of external recharging or refueling of devices. Enzymes such as multicopper oxidases (MCOs) belonging to oxidoreductase family can reduce oxygen into water performing oxygen reduction reaction (ORR). These reactions have been extensively studied and described in previous literature [14]. The most common "Blue" MCO enzymes are ascorbate oxidase (AOx), laccase ( $\mathrm{Lac})$, and bilirubin oxidase (BOx) that can act as excellent biocathode materials for biofuel cell applications [5, 6]. These enzymes are more active and selective than the state-of-the-art electrocatalyst platinum because BOx can promote a four-electron reduction of oxygen $[7,8]$, leading directly to water rather than production of significant amount of hydrogen peroxide $\left(\mathrm{H}_{2} \mathrm{O}_{2}\right.$ - 2-electron exchange) [9]. It has been shown previously that $\mathrm{BOx}$ demonstrated high electrocatalytic oxygen reduction and low overpotential necessary to catalyze the reaction and a turnover rate of 0.7 $\mathrm{O}_{2}$ per $\mathrm{Cu} \cdot \mathrm{s}^{-1}$, while Pt catalysts turnover rate is three times lower at overpotential of $350 \mathrm{mV}[2,10]$.

Moreover, BOx enzymes are well known to perform ORR when immobilized onto the surface of solid supports. Enhanced activity has been observed when functionalized with nanomaterials, since nanomaterials have been considered as excellent scaffolding structures for immobilization of the enzymes without sacrificing their bioactivity. The ORR is mainly dependent on the structure of $\mathrm{BOx}$ which was well 
studied and reported previously $[11,12]$. It contains three different copper centers (T1, T2, and T3) with overall four copper ions that catalyze the oxidation of bilirubin to biliverdin [13], thereby reducing molecular oxygen to water. The mechanism of electron transfer involves the T1 site of MCO acting as the primary electron acceptor from the substrate via an intramolecular electron transfer (IET) to the T2/T3 cluster site which converts molecular oxygen to water. Recent research unveiled whether the ORR in BOx is a four-electron transfer or a two-electron transfer with a hydrogen peroxide intermediate $[7,14]$. Much research has also been devoted in understanding the $\mathrm{BOx}$ direct electrochemistry (DET) and electron transfer via mediation by redox mediators [15-17]. Nevertheless, the use of these enzymes as biocatalysts has not yet been generally adopted for commercial purposes.

One persistent challenge is maintaining the catalytic activity of the enzyme and improving the performance often when immobilized on a solid support $[18,19]$. Enzymes may adsorb successfully and however tend to denature rendering some of the immobilized enzyme inactive and ineffective [20-22]. To overcome these challenges, enzymes that catalyze electron transfer reactions must be entrapped in hydrogels or stabilized with the use of orienting agents. Orienting agents promote correct and proper allocation of enzymes on the surface of the electrode to obtain high current density. The lone copper on $\mathrm{BOx}$ should not be no more than 1$2 \mathrm{~nm}$ from the electrode surface to avoid interfacial electron transfer being the rate-limiting step in oxygen reduction electrocatalysis [23, 24].

Recent studies have demonstrated a trend in surface modifications at biointerface level for MCOs based biocathode that incorporates the use of aromatic, hydrophobic, and hydrophilic molecules in order to suitably orient these redox enzymes with the $\mathrm{T} 1$ copper site immobilized on carbon nanotube sidewalls to enhance the oxygen electroreduction [25]. These molecules are natural substrates that are specific for the MCO enzymes for oxygen reduction. Laccase was initially studied by the Armstrong group where the hydrophobic pocket of laccase interaction with polycyclic aromatic compounds such as anthracene resulted in remarkable enhancement of electrocatalytic currents. The aromatic compounds structure is very similar to the natural substrate of Lac (phenols), and the strong hydrophobic interactions have been reported to promote the apt orientation for the DET [26]. Similar strategy was extended to the BOx enzymes where the studies show that the substrate-pocket did not exhibit hydrophobic interactions but electrostatic interactions, which are an efficient way to achieve direct wiring of $\mathrm{BOx}$ [27]. Along this line, different literature reports have focused on incorporating specific substrates of $\mathrm{BOx}$ such as bilirubin [28], quinones [29], and syringaldazine [30] towards appropriate orientation that can be convenient for DET-type electrocatalysis. Our group has previously reported on crosslinking the enzyme to the electrode with orienting agents, two bilirubin functional analogues, pyrrole2-carboxaldehyde and 2,5- dimethyl-1-phenyl-1H-pyrrole-3carbaldehyde, for enzyme orientation and 1-pyrenebutanoic acid, succinimidyl ester (PBSE) as the tethering agent. These compounds were chosen because they each contain a pyrrole moiety functionalized with a carbonyl group. Thus, the electronegative $\mathrm{N}$-atom from the pyrrole moiety and the $\mathrm{O}$ atom from the aldehyde group can act as hydrogen bond acceptors and the H-atom as a hydrogen bond donor [29]. Subsequently, we reported on the utilization of syringaldazine (Syr), for enzyme orientation, of both Laccase and BOx that demonstrated approximately 6 and 9 times increase in current density, respectively, compared to physically adsorbed and randomly oriented Lac cathodes [30].

Our present study follows from observations that the activity and the performance of the oriented $\mathrm{BOx}$ were further enhanced from incorporating a porphyrin precursor solution-hematin-on the biocathode. Hematin is a ferriprotoporphyrin-IX with a hydroxide ion bound to the ferric ion formed when hemin (ferriprotoporphyrin-IX with a chloride ion bound to ferric ion) is treated with strong $\mathrm{NaOH}$ solution. Hemin is an active center of family of hemeprotein, such as b-type cytochromes, peroxidase, myoglobin, and hemoglobin. The first electrochemical behavior of hemin was studied in 1968 on a platinum electrode by coulostatic method [31]. Hemin adsorption on graphite electrode demonstrated fast electron transfer that can exceed monolayer coverage with high amount of active species. Several literature studies show that hemin modified electrodes were extended in the catalysis and reduction of hydrogen peroxide [32-34], oxygen [35], and superoxide [31]. Utilization of hemin, protoporphyrin derivatives as pretreatment of the surface for improving the activity of BOx on cathode electrode has also been reported [36].

Herein, we demonstrate the enhancement of $\mathrm{BOx}$ air breathing cathode performance via the modification of the electrode with hematin. We also investigated the capability and effects of hematin versus hemin to promote an efficient electron transfer mechanisms for oxygen reduction. We further constructed a mediatorless and compartmentless glucose $/ \mathrm{O}_{2}$ DET-type biofuel cell to investigate the cell performance.

\section{Materials and Methodology}

Hemin, 1-pyrenebutanoic acid, succinimidyl ester (PBSE) was obtained from Setareh Biotech LLC; 1-ethyl-3-(3dimethylaminopropyl) carbodiimide hydrochloride (EDC) was obtained from TCI America; and N-hydroxysuccinimide $\pm 98 \%$ (NHS) was obtained from Alfa Aaesar. Hematin $(94.5 \%)$ was obtained from Chem-Impex Int'l Inc. NAD-dependent glucose dehydrogenase (GDH, from Pseudomonas sp., E.C. 1.1.1.47) and glucose were obtained from Sigma Aldrich and used as received; BOx was obtained from Myrothecium verrucaria (EC 1.3.3.5.), Amano Enzyme U.S.A. 50 Co., Ltd. Multiwalled nanotubes (MWNTs) paper (Buckeye Composites), MWNTs $(d=20-30 \mathrm{~nm}, L$ $=10-30 \mu \mathrm{m})$, and single walled nanotubes (SWNTs) $(99 \%$ purity) were obtained from cheaptubes.com.

\section{Electrode Preparations}

3.1. Hemin and Hematin Modified Electrodes. Air breathing cathodes were fabricated as in the previously described procedure with slight modification [37]. Briefly, teflonized carbon 


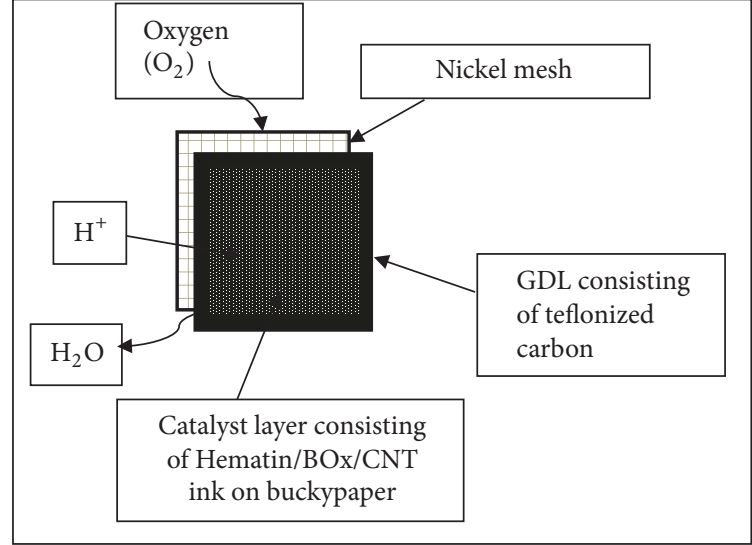

(a)

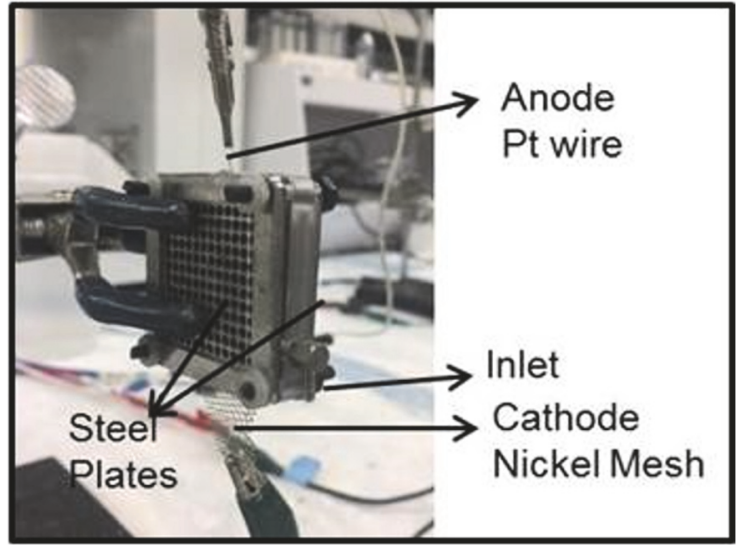

(b)

FIGURE 1: (a) Schematics of hematin-BOx air breathing cathode and (b) image of the biofuel cell.

black powder (35\% teflonization and 50\% teflonization, XC35 and XC50) and MWNTs paper (Buckeye Composites) were hydraulically pressed for 5 minutes at 500 psi. Figure 1 shows the schematic illustration of fabrication of gas diffusion layer (GDL) cathode (a) and image of the biofuel cell (b). A $10 \mathrm{mM}$ hemin solution was prepared by mixing hemin into DMSO. A $10 \mathrm{mM}$ hematin solution was prepared by mixing hemin into $20 \mathrm{mM}$ sodium hydroxide $(\mathrm{NaOH})$ solution. The $20 \mathrm{mM} \mathrm{NaOH}$ solution was also used to prepare cathodes with $\mathrm{NaOH}$ only modification. The as-prepared solutions were sonicated for 1 hour and $285 \mu \mathrm{L}$ of the mixture was deposited onto the buckeye paper layer $\left(2 \mathrm{~cm}^{2}\right)$ of the pressed air breathing cathode. The electrodes were left to dry for 4 hours at room temperature prior to being stored overnight at $4^{\circ} \mathrm{C}$.

3.2. Hemin and Hematin Modified Electrodes with BOx. Further investigation was carried out with BOx immobilized on hemin and hematin modified cathodes. Preparation of BOx ink was carried out as follows: $2 \mathrm{wt} \%$ MWNT ink solution was prepared by dissolving 4:1 ratio of water to ethanol (by weight) and added to $100 \mathrm{mg}$ MWNTs (10-20 nm). The prepared mixture was subjected to sonication for 1 hour in ice bath vortexing every $20 \mathrm{~min}$ interval time. $5 \mu \mathrm{L}$ of $300 \mathrm{mM}$ Di-Carb (in DMSO) and $170 \mu \mathrm{L}$ of stock solution of $2 \% \mathrm{MWNT}$ ink were mixed, vortexed, and incubated for 1 hour. This was followed by addition of $5 \mu \mathrm{L}$ of $300 \mathrm{mM}$ PBSE (in DMSO) and $20 \mathrm{uL}$ of water and left for incubation for 1 hour. To this, BOx ( $8 \mathrm{mg}, 16$ units $/ \mathrm{mL})$ was weighed and added to MWNT ink and incubated again for 1 hour at room temperature. Later $200 \mu \mathrm{L}$ of the ink was then deposited on the prewetted buckeye paper of air breathing cathode modified with hemin and hematin, respectively. Control electrodes were prepared by drop casting the ink onto unmodified air breathing cathodes. Following ink deposition, a chemical deposition of tetramethyl orthosilicate (TMOS) was performed by sealing cathodes in a Petri dish containing small caps filled with water and TMOS. The Petri dish remained sealed for $5 \mathrm{~min}$ before discarding the TMOS. Cathodes were then stored at $4^{\circ} \mathrm{C}$ overnight.
3.3. Hematin/EDC-NHS Coupling Modified Electrodes with $B O x$. The air breathing $\mathrm{BOx} /$ hematin electrodes were successively modified with additional components: 1-(3dimethylaminopropyl)-3' ethylcarbodiimide hydrochloride (EDC) and N-hydroxysuccinimide (NHS). Hematin solution $(10 \mathrm{mM})$ was prepared as described in previous section. After 1-hour sonication, $9.8 \mathrm{mg}$ of EDC and NHS was added and incubated to $285 \mu \mathrm{L}$ hematin solution. BOx ink described earlier was immobilized onto the modified hematin/EDCNHS air breathing cathodes were left for 4 hours at room temperature prior to being stored overnight at $4^{\circ} \mathrm{C}$. Figure 2 shows the schematic representation of stepwise procedure of hematin modification and BOx deposition on GDL cathode. Oxygen enters the cathode through the teflonized carbon GDL and is reduced to $\mathrm{H}_{2} \mathrm{O}$ at the hematin-BOx catalyst layer.

3.4. Electrochemical Testing. All electrochemical experiments were performed with a VMP3 potentiostat (Biologic). A conventional three-electrode system was used in the measurements with $\mathrm{Ag} / \mathrm{AgCl}$ as a reference electrode, a $\mathrm{Pt}$ wire as the auxiliary electrode, and a bare or modified electrode as working electrode. Cyclic voltammetry was carried out and potentiostatic polarization curves were obtained to characterize half-cell testing. All tests were conducted in $245 \mathrm{mM}$, pH 7.0 PBS buffer at room temperature.

3.5. Complete Fuel Cell Testing. Glucose dehydrogenase (GDH, Toyobo) based anodes were prepared for complete fuel cell testing. Polymethylene green (PMG) was electrodeposited onto (electrode area $7.3 \mathrm{~cm}^{2}$ ) carbon felt electrodes following a modified version of a previously described procedure [30]. A single wall nanotube (SWNT-PEI) ink solution containing GDH was drop-casted on top of the PMG treated carbon felt electrodes. The anodes were stored overnight at $4^{\circ} \mathrm{C}$ prior to testing. The GDH anodes were paired with $9 \mathrm{~cm}^{2}\left(7.3 \mathrm{~cm}^{2}\right.$ working area) hematin modified $\mathrm{BOx}$ or unmodified $\mathrm{BOx}$ cathodes for complete fuel cell testing. The fuel cells were tested with $100 \mathrm{mM}$ glucose $/ 10 \mathrm{mM}$ nicotinamide adenine dinucleotide (NAD) in $245 \mathrm{mM}$ PBS buffer pH 7.0. A Constant Load Discharge (CLD) technique 

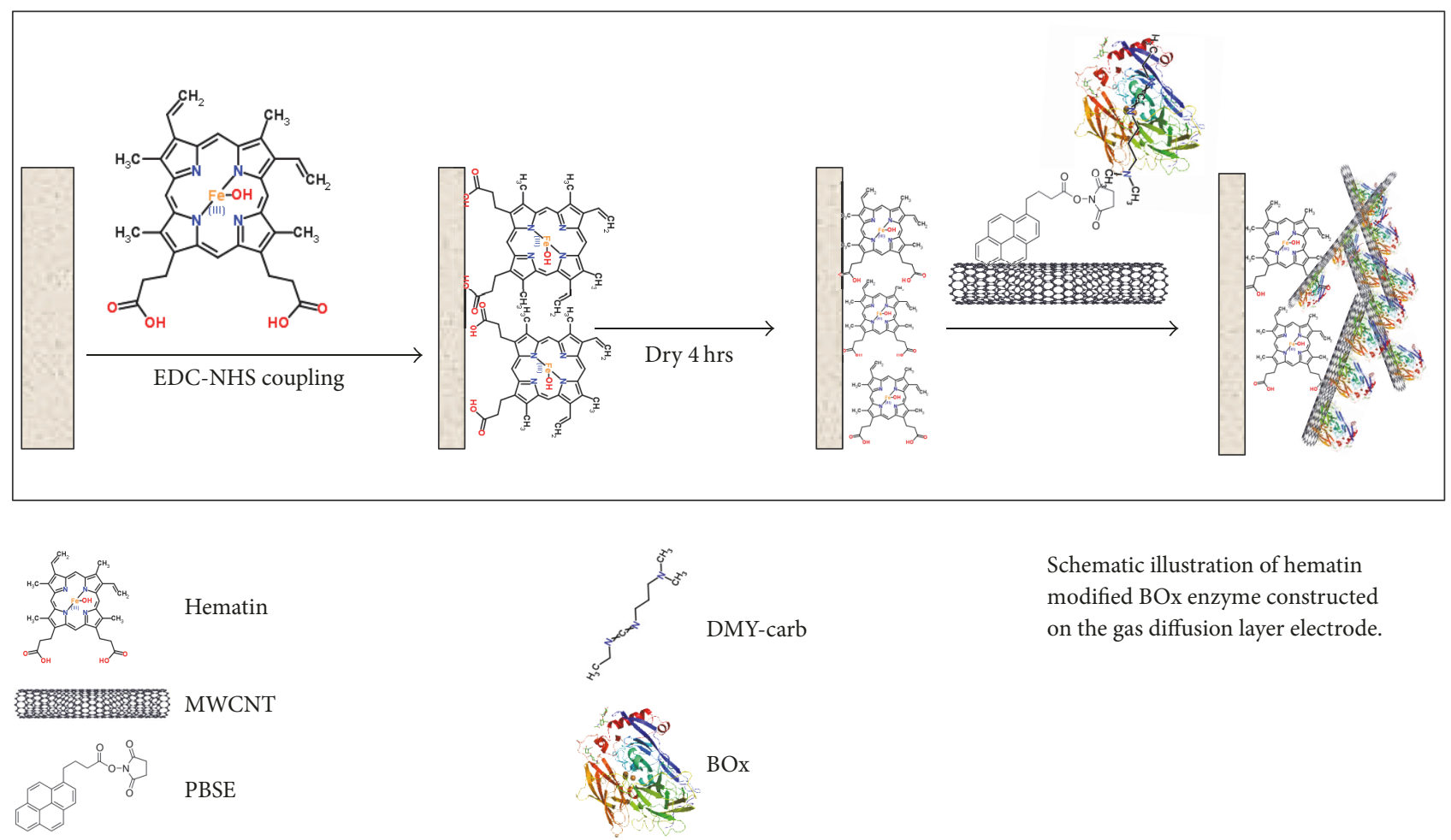

Schematic illustration of hematin modified BOx enzyme constructed on the gas diffusion layer electrode.

FIGURE 2: Schematic illustration of stepwise procedure of hematin modified BOx on gas diffusion layer cathode.

was employed to generate power and current density curves. Later, discharge curves were generated by applying a constant load of $3.0 \mathrm{~mA}$ to the fuel cell. The cells were filled with fresh fuel before each subsequent discharge. Preparation of $\mathrm{BOx}$ ink for complete fuel cell testing was described in Supplementary File.

\section{Results and Discussion}

4.1. Electrochemical Testing and Characterization of Hemin and Hematin Electrodes. To better realize the function towards the enhancement of BOx cathode, hematin air breathing cathodes were prepared without $\mathrm{BOx}$ to determine if the electrodes were independently capable of catalyzing the ORR. This test was compared simultaneously with hemin air breathing cathode without BOx. The step potential studies were conducted to evaluate the electrodes under quiescent with no oxygen saturation conditions. At $50 \mathrm{mV}$, the current density for the hemin cathode was $0.4 \mathrm{~mA} / \mathrm{cm}^{2}$ (Figure 3) while current density of $0.05 \mathrm{~mA} / \mathrm{cm}^{2}$ was observed for hematin. The results showed that hemin demonstrated higher current density compared to hematin suggesting that hemin can serve as a catalyst for ORR. Several studies have identified hemin as a DET-type electrocatalyst for ORR. Ma et al. have shown that hemin modified PAMAM/MWCNT nanocomposite films on glassy carbon electrodes can act as both an electron conductor and catalytic mediator for L-tyrosine [38]. Others have identified hemin as an electrocatalyst for oxygen reduction and superoxide detection biosensors. Therefore, the increase in current density was predictable; however very little increase in current density was observed for the hematin electrodes. This suggests that hematin without the

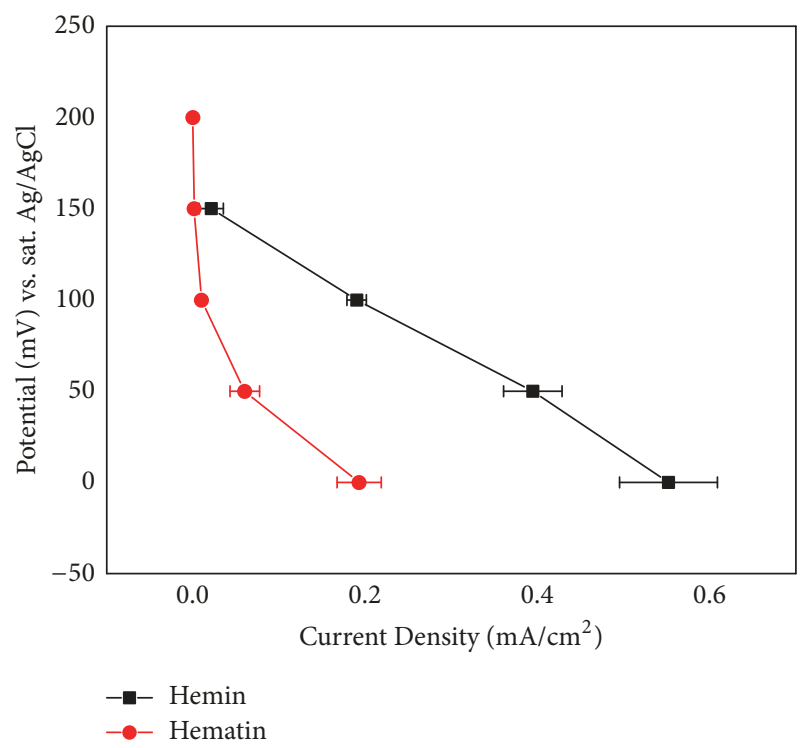

FIgURE 3: Representative step potential curves for hemin (black square) and hematin (red circle) air breathing cathodes tested with 245 mM PBS, pH 7.0.

BOx does not act as an electrocatalyst by itself and cannot assist as a catalytic mediator for ORR reduction. Therefore, we may assume that hematin adsorbed onto $\mathrm{BOx}$ cathodes may enhance DET catalysis by acting as a natural substrate or precursor for the BOx enzyme.

A cyclic voltammetry (CV) study was conducted to compare the electrochemical behavior of hematin modified air breathing cathodes with bare and EDC/NHS-hematin 
modified cathodes. Figure 1 (Supplementary) shows that the hematin modified air breathing cathodes had a reduction peak at around $300 \mathrm{mV}$ when compared to a bare air breathing cathode. The peak current density was $3 \mathrm{~mA} / \mathrm{cm}^{2}$ while no clear oxidation peaks have been observed. Since no distinct reversible redox reaction was observed for the hematin modified air breathing cathodes, hematin most likely acts as a natural substrate and a precursor for oxygen reduction reaction (ORR) at the $\mathrm{BOx}$ cathode. In literature, hematin has been identified as a potential alternative to horseradish peroxidase (HRP) for $\mathrm{H}_{2} \mathrm{O}_{2}$ detection [39] and catalysis of phenol compounds [40]. With further addition of crosslinking couple EDC/NHS, the reduction peak for the hematin modified electrodes has become less distinct. A high scan rate of $250 \mathrm{mV} / \mathrm{s}$ was employed for the $\mathrm{CV}$ study to test the stability of the EDC/NHS-hematin air breathing cathodes. Figure 2 (Supplementary) compares cycle 2 to cycle 20 for the air breathing cathodes modified with EDC/NHS-hematin. There was little variation observed between the magnitude of cycles 2 and 20. Shrinking CV curves would suggest that the EDC/NHS-hematin was being stripped from the electrode surface. The result of this study suggests that the air breathing cathodes modified with hematin/EDC/NHS were stable. The stability of the EDC/NHS-hematin electrodes was most likely due to the covalent attachment of the hematin to $\mathrm{BOx}$ via the EDC/NHS crosslinker. Hematin contains two carboxylic acid moieties, which allows the hematin to react with EDC and NHS further stabilizes the EDC-hematin intermediate and allows the intermediate product to form a covalent attachment to the BOx. EDC and NHS promote the reaction of carboxylic groups of hematin with amino groups of the BOx enzyme.

\subsection{Electrochemical Testing and Characterization of Hemin/} $B O x$ and Hematin/BOx Electrodes. We further examined the effect of hematin, hemin, and nonmodified BOx catalyzed oxygen reduction. A comparison of the current densities from potentiostatic polarization curves is shown in Figure 4. The current density from the electrode containing BOx and hemin was $0.007 \mathrm{~mA} / \mathrm{cm}^{2}$. The current density for BOx only was $0.32 \mathrm{~mA} / \mathrm{cm}^{2}$, and the current density in the presence of hematin modified was $0.7 \mathrm{~mA} / \mathrm{cm}^{2}$. For all electrodes the current densities generated at $300 \mathrm{mV}$ versus sat $\mathrm{Ag} / \mathrm{AgCl}$ concluded that hematin led to a significant increase in $\mathrm{BOx}$ performance. The polarization experiments with hematin demonstrated more than 2 times increase in the maximum current densities compared to only BOx cathodes with no enhancements (Figure 4). Hematin is a ferriprotoporphyrinIX with a hydroxide ion bound to the ferric ion formed when hemin (ferriprotoporphyrin-IX with a chloride ion bound to ferric ion) is treated with strong $\mathrm{NaOH}$ solution. As mentioned previously, hemin has been studied as direct electron transfer moiety for oxygen reduction. Therefore, the function of hematin as an enhancement agent was compared with hemin and ferricyanide as electron transfer mediators (data not shown). Many such hemin related compounds have been identified in literature. Catechol, hydroquinone, pyrogallol, and bilirubin which are catalytically oxidized by BOx were employed in modification of biocathodes [28]. Recent studies

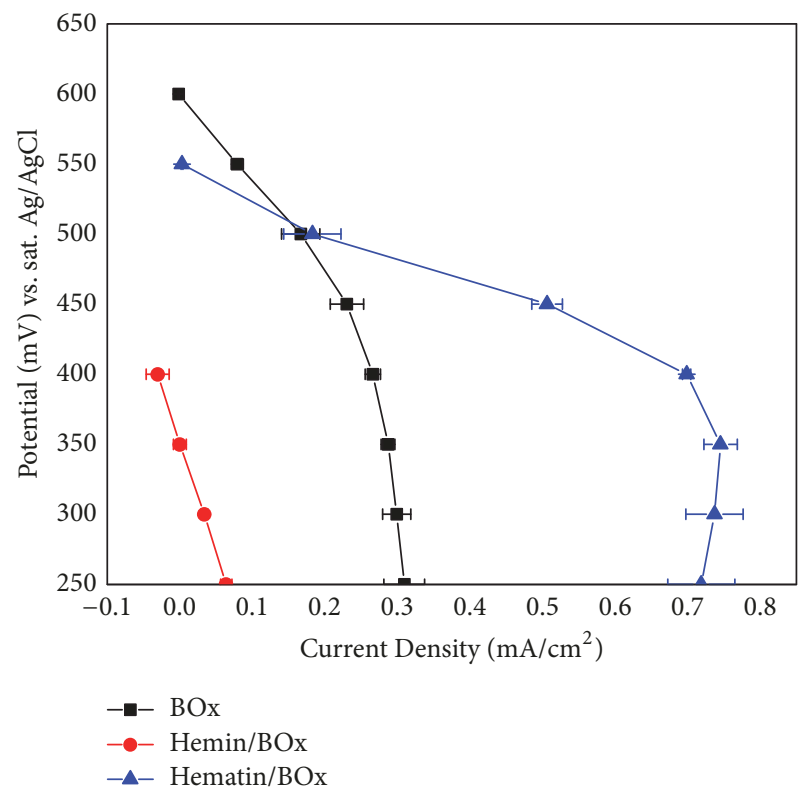

FIGURE 4: Representative step potential curves for BOx (black square), hemin-BOx (red circle), and hematin-BOx (blue triangle) air breathing cathodes tested in $245 \mathrm{mM}$ PBS, pH 7.0.

have focused on incorporating specific substrates of BOx such as bilirubin, quinones, syringaldazine, and protoporphyrin derivatives such as protoporphyrin IX iron (III) (PPFeIII), protoporphyrin IX dimethyl ester (PPDE), and octaethylporphyrin (OEP) towards appropriate orientation that can be convenient for DET-type electrocatalysis [36]. However no study has been previously done on hematin as substrate for BOx. Therefore, hematin was further tested as a precursor and premodifier in the enhancement of BOx enzyme.

To demonstrate that improved performance was due to the addition of hematin, BOx electrodes were tested with only $\mathrm{NaOH}$ modification, $\mathrm{NaOH}$-hematin, and $\mathrm{NaOH}$ EDC/NHS-hematin. Based on the results (Figure 5), treating the $\mathrm{BOx}$ electrode with $\mathrm{NaOH}$ led to enhanced air breathing cathode performance. However, performance was higher when the electrodes were modified with hemin in $\mathrm{NaOH}$. Increased current densities achieved with $\mathrm{NaOH}$ modification were most likely due to the electrode surface becoming more hydrophilic, which would create a more favorable environment for the ORR taking place at the electrode surface. The highest current densities were achieved when the crosslinking couple EDC/NHS were added to the hematin solution. Current densities greater than $0.8 \mathrm{~mA} / \mathrm{cm}^{2}$ were achieved by modifying the electrode surface with the EDC/NHS-hematin solution. All BOx cathode types were tested in triplicate. The EDC/NHS BOx air breathing cathodes performed better than the BOx only (no enhancements) cathodes at $300 \mathrm{mV}$ but performed significantly less than the $\mathrm{NaOH}, \mathrm{NaOH}$-hematin, and $\mathrm{NaOH}$ (EDC/NHS)-hematinBOx cathodes. The $\mathrm{NaOH}$ (EDC/NHS)-hematin-BOx cathodes remained the highest performing cathodes.

4.3. BOx Loading Optimization. High enzyme loadings are required for the optimal fuel cell performance. However, 


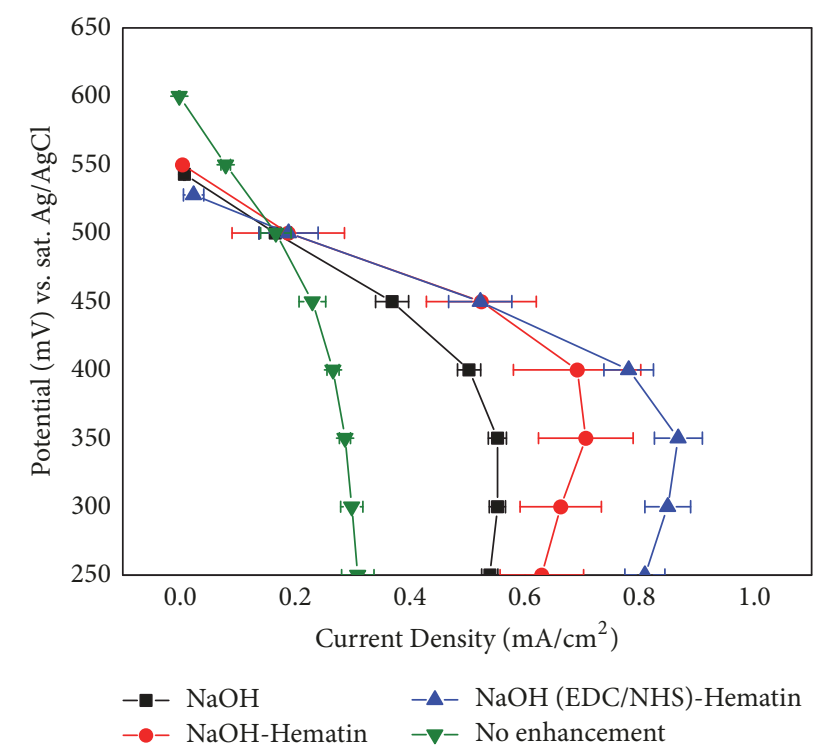

FIGURE 5: Representative step potential curves for $\mathrm{BOx}$ air breathing cathodes modified with $\mathrm{NaOH}$ only (black square), $\mathrm{NaOH}$-hematin (red circle), and $\mathrm{NaOH}$ (EDC/NHS)-hematin (blue triangle) or no enhancement (green inverted triangle) tested in $245 \mathrm{mM}$ PBS, $\mathrm{pH}$ 7.0.

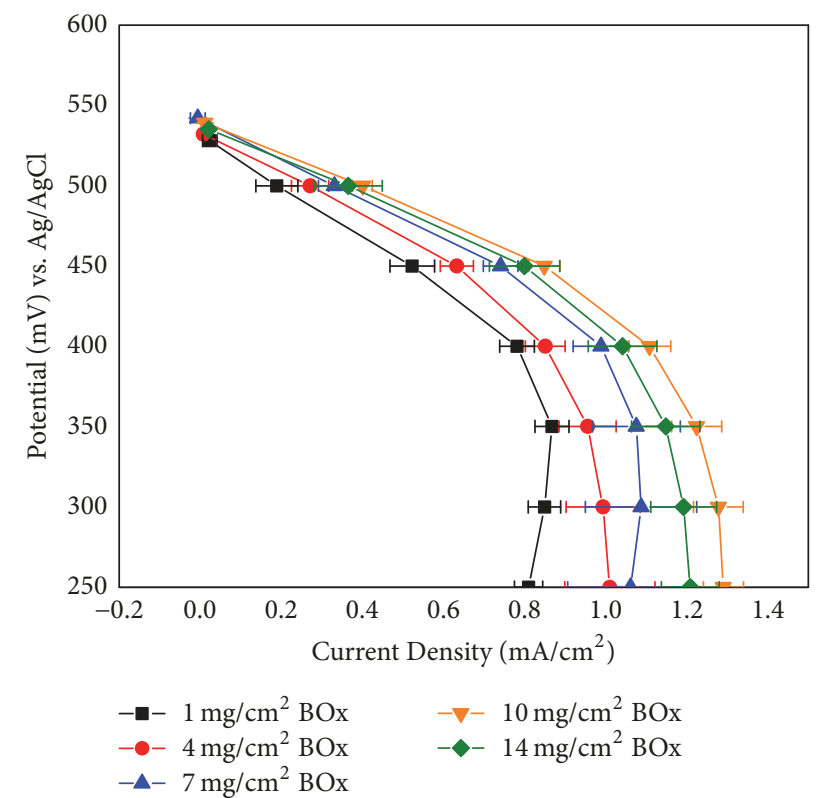

FIGURE 6: Representative step potential curves for BOx air breathing cathodes prepared with different amounts of $\mathrm{BOx}$ ranging from $1 \mathrm{mg} / \mathrm{cm}^{2}$ to $14 \mathrm{mg} / \mathrm{cm}^{2}$ tested in $245 \mathrm{mM}$ PBS, $\mathrm{pH}$ 7.0.

when the enzyme loadings are too high, substrate diffusion and product inhibition can limit the rate of overall processes. To minimize the mass diffusion limitations, the BOx loading on hematin modified air breathing cathodes was optimized. The enzyme loading ranging from $1 \mathrm{mg} / \mathrm{cm}^{2}$ to $14 \mathrm{mg} / \mathrm{cm}^{2}$ was tested (Figure 6). The results show increase in current densities with increase in BOx loading on the cathode. Since the initial rate of reaction of the enzyme corresponds to the activity and amount of enzyme loaded, the current

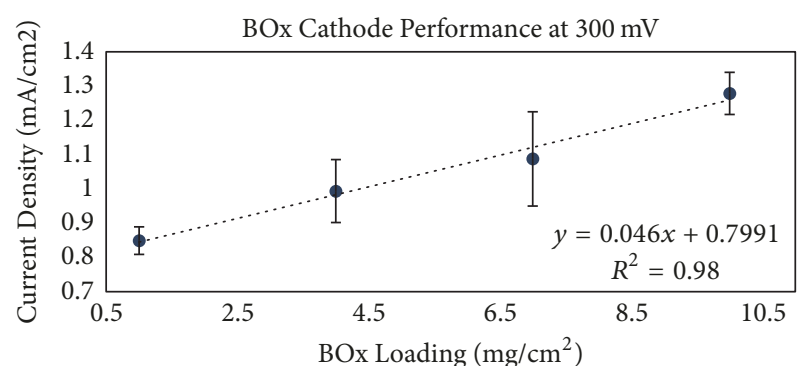

FIGURE 7: Linear regression plot for current density as a function of BOx loading.

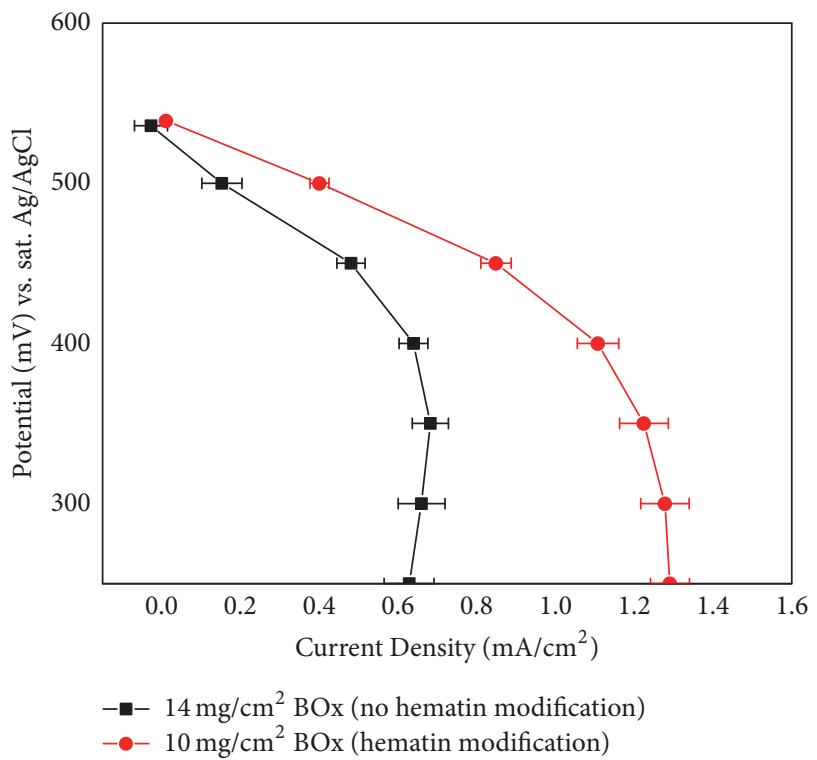

FIGURE 8: Representative step potential curves for optimized BOx air breathing cathodes prepared without hematin (black square) or with hematin (red circle) tested in 245 mM PBS, pH 7.0.

increased until optimal loading of $10 \mathrm{mg} / \mathrm{cm}^{2}$ has reached and decreased when the electrode surface was loaded with $14 \mathrm{mg} / \mathrm{cm}^{2}$ enzyme. This could be due to diffusion limitations where part of the immobilized enzyme may be not being available for catalysis. Figure 7 shows the linear dependence of current density at $300 \mathrm{mV}$ with the $\mathrm{BOx}$ loading ranging from $1 \mathrm{mg} / \mathrm{cm}^{2}$ to $10 \mathrm{mg} / \mathrm{cm}^{2}$. It is to be noted that nonhematin-BOx cathodes performed optimally at $14 \mathrm{mg} / \mathrm{cm}^{2}$ BOx loading and by employing hematin, BOx loading was reduced by $29 \%$. At $300 \mathrm{mV}$, the optimized hematin-BOx cathode performed twice better than the non-hematin-BOx cathode (Figure 8).

4.4. Complete Fuel Cell Testing. A DET-type biofuel cell without separators was constructed. The biocathode was an air breathing hematin/BOx and the bioanode was GDH/PMG carbon felt electrodes. Complete fuel cell testing was conducted with $100 \mathrm{mM}$ glucose/10 mM NAD fuel in $245 \mathrm{mM}$ PBS buffer $\mathrm{pH} 7.0$ under quiescent conditions at room temperature. The current densities dependence of the cell power density is shown in Figure 9(a) while Figure 9(b) shows 


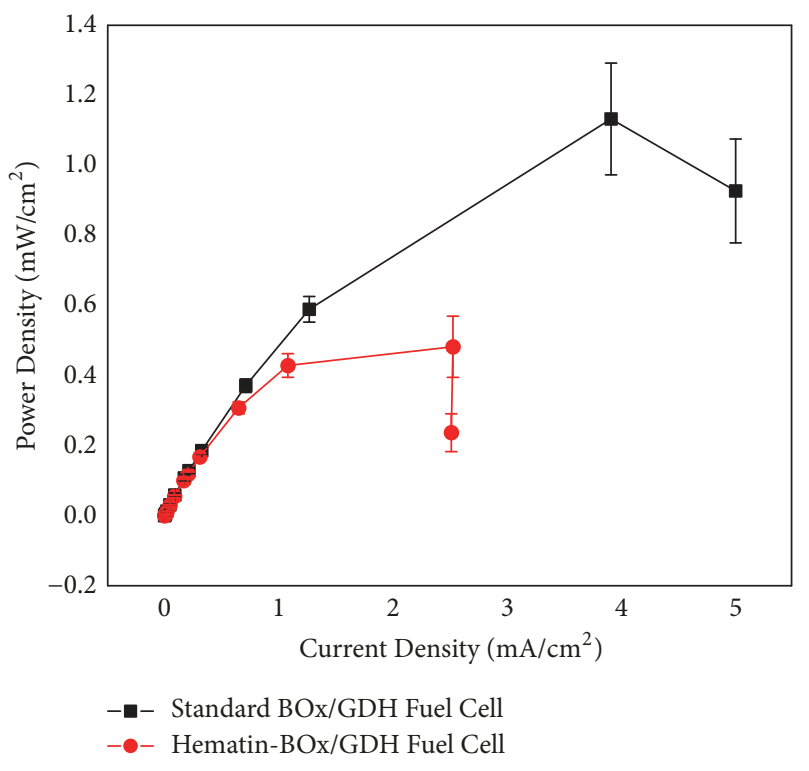

(a)

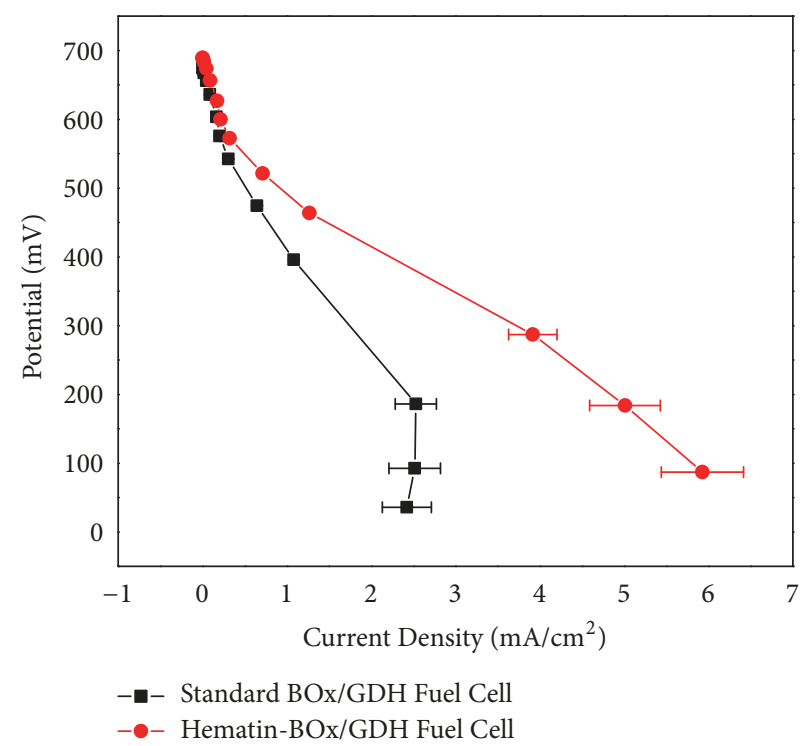

(b)

FIGURE 9: Representative power density (a) and current density (b) curves for optimized BOx air breathing cathodes prepared without hematin (black square) or with hematin (red circle) paired with GDH anodes tested in $100 \mathrm{mM}$ glucose.

the maximum current densities for the hematin modified BOx cathodes. The open circuit potential of $0.7 \mathrm{~V}$ was obtained. The maximum current density was $6.6 \mathrm{~mA} / \mathrm{cm}^{2}$ and maximum power density was $1.1 \mathrm{~mW} / \mathrm{cm}^{2}$ at $300 \mathrm{mV}$ of the fuel cell. On the other hand, with non-hematin modified cathode the maximum current density of $2.5 \mathrm{~mA} / \mathrm{cm}^{2}$ and maximum power density of $0.2 \mathrm{~mW} / \mathrm{cm}^{2}$ were obtained. From the results the determining factor for the high current density is controlled by the BOx cathode. The performance of the hematin-BOx cathodes was 2.4 times higher than the performance for the $\mathrm{BOx}$ cathodes without hematin modification. Constant current load discharge studies of the hematin/BOx cathode and GDH anodes based biofuel cells were further conducted with $50 \mathrm{mM}$ glucose $/ 10 \mathrm{mM}$ NAD fuel in $245 \mathrm{mM}$ PBS buffer $\mathrm{pH}$ 7.0. A constant current load of $3.0 \mathrm{~mA}$ was applied to each fuel cell until the potential reached $350 \mathrm{mV}$. With injection of fresh fuel, the fuel cell was tested under repeated discharge cycles. A total of 4 discharges were performed consecutively with 10-minute recovery periods between discharges as shown in Figure 3 (Supplementary). The average runtime varied between $0.76 \mathrm{~h}$ and $1.0 \mathrm{~h}$ with average capacity ranging from $126 \mathrm{mAh} / \mathrm{g}$ to $167 \mathrm{mAh} / \mathrm{g}$. The capacity of the BOx/GDH fuel cells decreased by less than $25 \%$ after being discharged to $350 \mathrm{mV}$ multiple times. The results suggest that the fuel cells can withstand multiple discharges (with fresh fuel) without experiencing a significant loss in capacity. These results also indicate that there was minimal leaching out of adsorbed functionalities of anode and cathode biomaterials.

\section{Conclusion}

In this study, direct interaction of BOx on hematin modified MWCNT is capable of increasing the current density driven by a combination of synchronous effect of natural substrate and favorable orientation for BOx. With hematin based electrode surface modification the optimized loading of BOx was drastically decreased by $28.6 \%$, and an average peak power density above $1 \mathrm{~mW} / \mathrm{cm}^{2}$ was achieved. Using hematin, it was investigated that the assembly of hematin with EDC/NHS resulted in significant increase of current density compared to the non-hematin modified electrode. The hematin modified electrode showed remarkable stability when subjected to more than 15 cycles of cyclic voltammetry. The current density as high as $6.6 \mathrm{~mA} / \mathrm{cm}^{2}$ at $300 \mathrm{mV}$ and power density of $1.1 \mathrm{~mW} / \mathrm{cm}^{2}$ were obtained with $\mathrm{GDH} / \mathrm{O}_{2}$ biofuel cell.

\section{Disclosure}

The content of this manuscript does not necessarily reflect the position or the policy of the Government, and no official endorsement should be inferred. Abstract on this work was submitted at $233^{\text {rd }}$ ECS Meeting.

\section{Conflicts of Interest}

The authors declare no conflicts of interest.

\section{Acknowledgments}

This research was supported by the U.S. Army Research Office (STTR Contract W911NF-13-C-0015).

\section{Supplementary Materials}

Supplementary materials contain the procedure for preparation of bilirubin oxidase (BOx) ink for complete fuel 
cell testing. The cyclic voltammetry studies for testing the bare, hematin modified, and EDC/NHS-hematin modified electrode are shown in Supplemental Figure 1 and showed the stability of hematin modified electrode (Supplemental Figure 2). Recycling and discharge studies under constant current load of GDH/BOx fuel cell were shown in Supplemental Figure 3. (Supplementary Materials)

\section{References}

[1] N. Mano and L. Edembe, "Bilirubin oxidases in bioelectrochemistry: Features and recent findings," Biosensors and Bioelectronics, vol. 50, pp. 478-485, 2013.

[2] N. Mano, J. L. Fernandez, Y. Kim, W. Shin, A. J. Bard, and A. Heller, "Oxygen Is Electroreduced to Water on a "Wired" Enzyme Electrode at a Lesser Overpotential than on Platinum," Journal of the American Chemical Society, vol. 125, no. 50, pp. 15290-15291, 2003.

[3] N. Mano, H.-H. Kim, and A. Heller, "On the relationship between the characteristics of bilirubin oxidases and $\mathrm{O}_{2}$ cathodes based on their "wiring", "The Journal of Physical Chemistry $B$, vol. 106, no. 34, pp. 8842-8848, 2002.

[4] N. Mano, V. Soukharev, and A. Heller, "A laccase-wiring redox hydrogel for efficient catalysis of $\mathrm{O}_{2}$ electroreduction," The Journal of Physical Chemistry B, vol. 110, no. 23, pp. 11180-11187, 2006.

[5] E. I. Solomon, A. J. Augustine, and J. Yoon, "O $\mathrm{O}_{2}$ Reduction to $\mathrm{H}_{2} \mathrm{O}$ by the multicopper oxidases," Dalton Transactions, no. 30 , pp. 3921-3932, 2008.

[6] E. I. Solomon, U. M. Sundaram, and T. E. Machonkin, "Multicopper oxidases and oxygenases," Chemical Reviews, vol. 96, no. 7, pp. 2563-2606, 1996.

[7] S. Brocato, C. Lau, and P. Atanassov, "Mechanistic study of direct electron transfer in bilirubin oxidase," Electrochimica Acta, vol. 61, pp. 44-49, 2012.

[8] D. Ivnitski, K. Artyushkova, and P. Atanassov, "Surface characterization and direct electrochemistry of redox copper centers of bilirubin oxidase from fungi Myrothecium verrucaria," Bioelectrochemistry, vol. 74, no. 1, pp. 101-110, 2008.

[9] R. D. Milton, F. Giroud, A. E. Thumser, S. D. Minteer, and R. C. T. Slade, "Bilirubin oxidase bioelectrocatalytic cathodes: The impact of hydrogen peroxide," Chemical Communications, vol. 50, no. 1, pp. 94-96, 2014.

[10] C. C. L. McCrory, X. Ottenwaelder, T. D. P. Stack, and C. E. D. Chidsey, "Kinetic and mechanistic studies of the electrocatalytic reduction of $\mathrm{O}_{2}$ to $\mathrm{H}_{2} \mathrm{O}$ with mononuclear $\mathrm{Cu}$ complexes of substituted 1,10-phenanthrolines," The Journal of Physical Chemistry A, vol. 111, no. 49, pp. 12641-12650, 2007.

[11] B. T. Doumas, B. Perry, B. Jendrzejczak, and L. Davis, "Measurement of direct bilirubin by use of bilirubin oxidase," Clinical Chemistry, vol. 33, no. 8, pp. 1349-1353, 1987.

[12] E. I. Solomon, R. K. Szilagyi, S. DeBeer George, and L. Basumallick, "Electronic Structures of Metal Sites in Proteins and Models: Contributions to Function in Blue Copper Proteins," Chemical Reviews, vol. 104, no. 2, pp. 419-458, 2004.

[13] S. Murao and N. Tanaka, "A New Enzyme "Bilirubin Oxidase" Produced by Myrothecium verrucaria MT-1," Agricultural and Biological Chemistry, vol. 45, no. 10, pp. 2383-2384, 1981.

[14] L. Quintanar, C. Stoj, A. B. Taylor, P. J. Hart, D. J. Kosman, and E. I. Solomon, "Shall we dance? How a multicopper oxidase chooses its electron transfer partner," Accounts of Chemical Research, vol. 40, no. 6, pp. 445-452, 2007.

[15] P. Ramírez, N. Mano, R. Andreu et al., "Direct electron transfer from graphite and functionalized gold electrodes to $\mathrm{T} 1$ and T2/T3 copper centers of bilirubin oxidase," Biochimica et Biophysica Acta (BBA) - Bioenergetics, vol. 1777, no. 10, pp. 13641369, 2008.

[16] S. Shleev, A. El Kasmi, T. Ruzgas, and L. Gorton, "Direct heterogeneous electron transfer reactions of bilirubin oxidase at a spectrographic graphite electrode," Electrochemistry Communications, vol. 6, no. 9, pp. 934-939, 2004.

[17] S. Tsujimura, K. Kano, and T. Ikeda, "Bilirubin oxidase in multiple layers catalyzes four-electron reduction of dioxygen to water without redox mediators," Journal of Electroanalytical Chemistry, vol. 576, no. 1, pp. 113-120, 2005.

[18] M. Rabe, D. Verdes, and S. Seeger, "Understanding protein adsorption phenomena at solid surfaces," Advances in Colloid and Interface Science, vol. 162, no. 1-2, pp. 87-106, 2011.

[19] M. Rasmussen, S. Abdellaoui, and S. D. Minteer, "Enzymatic biofuel cells: 30 years of critical advancements," Biosensors and Bioelectronics, vol. 76, pp. 91-102, 2016.

[20] W. Norde, "Adsorption of proteins from solution at the solidliquid interface," Advances in Colloid and Interface Science, vol. 25, no. C, pp. 267-340, 1986.

[21] W. Norde and T. Zoungrana, "Surface-induced changes in the structure and activity of enzymes physically immobilized at solid/liquid interfaces," Biotechnology and Applied Biochemistry, vol. 28, no. 2, pp. 133-143, 1998.

[22] M. van der Veen, M. C. Stuart, and W. Norde, "Spreading of proteins and its effect on adsorption and desorption kinetics," Colloids and Surfaces B: Biointerfaces, vol. 54, no. 2, pp. 136-142, 2007.

[23] C. C. Page, C. C. Moser, X. Chen, and P. L. Dutton, "Natural engineering principles of electron tunnelling in biological oxidation-reduction," Nature, vol. 402, no. 6757, pp. 47-52, 1999.

[24] H. B. Gray and J. R. Winkler, "Long-range electron transfer," Proceedings of the National Acadamy of Sciences of the United States of America, vol. 102, no. 10, pp. 3534-3539, 2005.

[25] N. Lalaoui, A. L. Goff, M. Holzinger, M. Mermoux, and S. Cosnier, "Wiring laccase on covalently modified graphene: Carbon nanotube assemblies for the direct bio-electrocatalytic reduction of oxygen," Chemistry - A European Journal, vol. 21, no. 8, pp. 3198-3201, 2015.

[26] C. F. Blanford, C. E. Foster, R. S. Heath, and F. A. Armstrong, "Efficient electrocatalytic oxygen reduction by the 'blue' copper oxidase, laccase, directly attached to chemically modified carbons," Faraday Discussions, vol. 140, pp. 319-335, 2008.

[27] L. Dos Santos, V. Climent, C. F. Blanford, and F. A. Armstrong, "Mechanistic studies of the 'blue' $\mathrm{Cu}$ enzyme, bilirubin oxidase, as a highly efficient electrocatalyst for the oxygen reduction reaction," Physical Chemistry Chemical Physics, vol. 12, no. 42, pp. 13962-13974, 2010.

[28] K. So, S. Kawai, Y. Hamano et al., "Improvement of a direct electron transfer-type fructose/dioxygen biofuel cell with a substrate-modified biocathode," Physical Chemistry Chemical Physics, vol. 16, no. 10, pp. 4823-4829, 2014.

[29] R. J. Lopez, S. Babanova, Y. Ulyanova, S. Singhal, and P. Atanassov, "Improved interfacial electron transfer in modified bilirubin oxidase biocathodes," ChemElectroChem, vol. 1, no. 1, Article ID CELC201300085, 2014. 
[30] Y. Ulyanova, S. Babanova, E. Pinchon, I. Matanovic, S. Singhal, and P. Atanassov, "Effect of enzymatic orientation through the use of syringaldazine molecules on multiple multi-copper oxidase enzymes," Physical Chemistry Chemical Physics, vol. 16, no. 26, pp. 13367-13375, 2014.

[31] J. Chen, U. Wollenberger, F. Lisdat, B. Ge, and F. W. Scheller, "Superoxide sensor based on hemin modified electrode," Sensors and Actuators B: Chemical, vol. 70, no. 1-3, pp. 115-120, 2000.

[32] J. Chen, L. Zhao, H. Bai, and G. Shi, "Electrochemical detection of dioxygen and hydrogen peroxide by hemin immobilized on chemically converted graphene," Journal of Electroanalytical Chemistry, vol. 657, no. 1-2, pp. 34-38, 2011.

[33] G.-X. Wang, Y. Zhou, M. Wang, W.-J. Bao, K. Wang, and X.H. Xia, "Structure orientation of hemin self-assembly layer determining the direct electron transfer reaction," Chemical Communications, vol. 51, no. 4, pp. 689-692, 2015.

[34] Y. Guo, J. Li, and S. Dong, "Hemin functionalized graphene nanosheets-based dual biosensor platforms for hydrogen peroxide and glucose," Sensors and Actuators B: Chemical, vol. 160, no. 1, pp. 295-300, 2011.

[35] S. Antoniadou, A. D. Jannakoudakis, and E. Theodoridou, "Electrocatalytic reactions on carbon fibre electrodes modified by hemine II. Electro-oxidation of hydrazine," Synthetic Metals, vol. 30, no. 3, pp. 295-304, 1989.

[36] N. Lalaoui, A. Le Goff, M. Holzinger, and S. Cosnier, "Fully Oriented Bilirubin Oxidase on Porphyrin-Functionalized Carbon Nanotube Electrodes for Electrocatalytic Oxygen Reduction," Chemistry - A European Journal, vol. 21, no. 47, pp. 16868-16873, 2015.

[37] S. Babanova, K. Artyushkova, Y. Ulyanova, S. Singhal, and P. Atanassov, "Design of experiments and principal component analysis as approaches for enhancing performance of gasdiffusional air-breathing bilirubin oxidase cathode," Journal of Power Sources, vol. 245, pp. 389-397, 2014.

[38] Q. Ma, S. Ai, H. Yin, Q. Chen, and T. Tang, “Towards the conception of an amperometric sensor of l-tyrosine based on Hemin/PAMAM/MWCNT modified glassy carbon electrode," Electrochimica Acta, vol. 55, no. 22, pp. 6687-6694, 2010.

[39] S. Pirillo, F. S. García Einschlag, E. H. Rueda, and M. L. Ferreira, "Horseradish peroxidase and hematin as biocatalysts for alizarin degradation using hydrogen peroxide," Industrial \& Engineering Chemistry Research, vol. 49, no. 15, pp. 6745-6752, 2010.

[40] J. A. Akkara, J. Wang, D.-P. Yang, and K. E. Gonsalves, "Hematin-catalyzed polymerization of phenol compounds," Macromolecules, vol. 33, no. 7, pp. 2377-2382, 2000. 

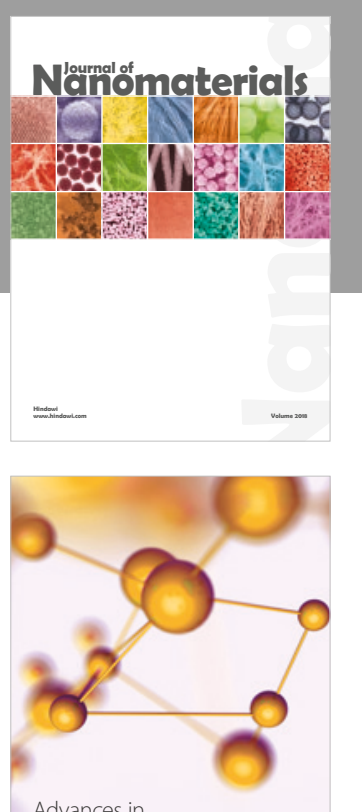

Physical Chemistry
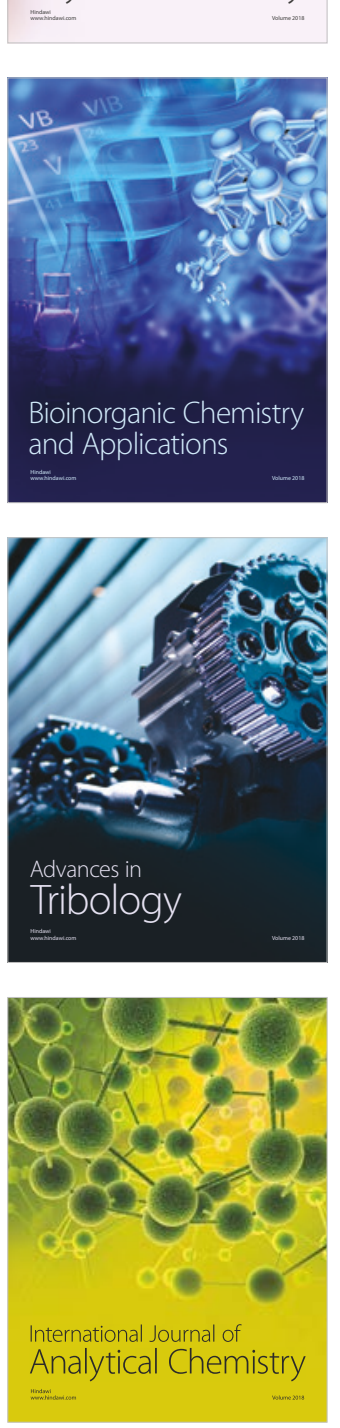

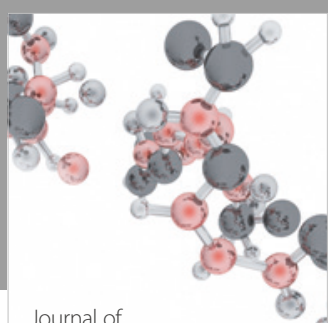

Analytical Methods

in Chemistry

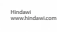

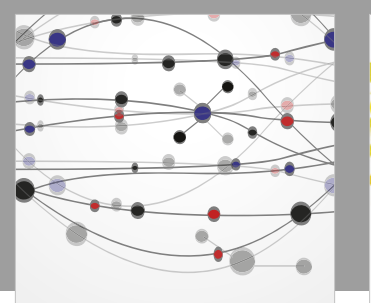

The Scientific World Journal

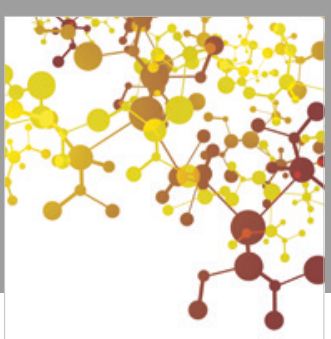

Journal of

Applied Chemistry
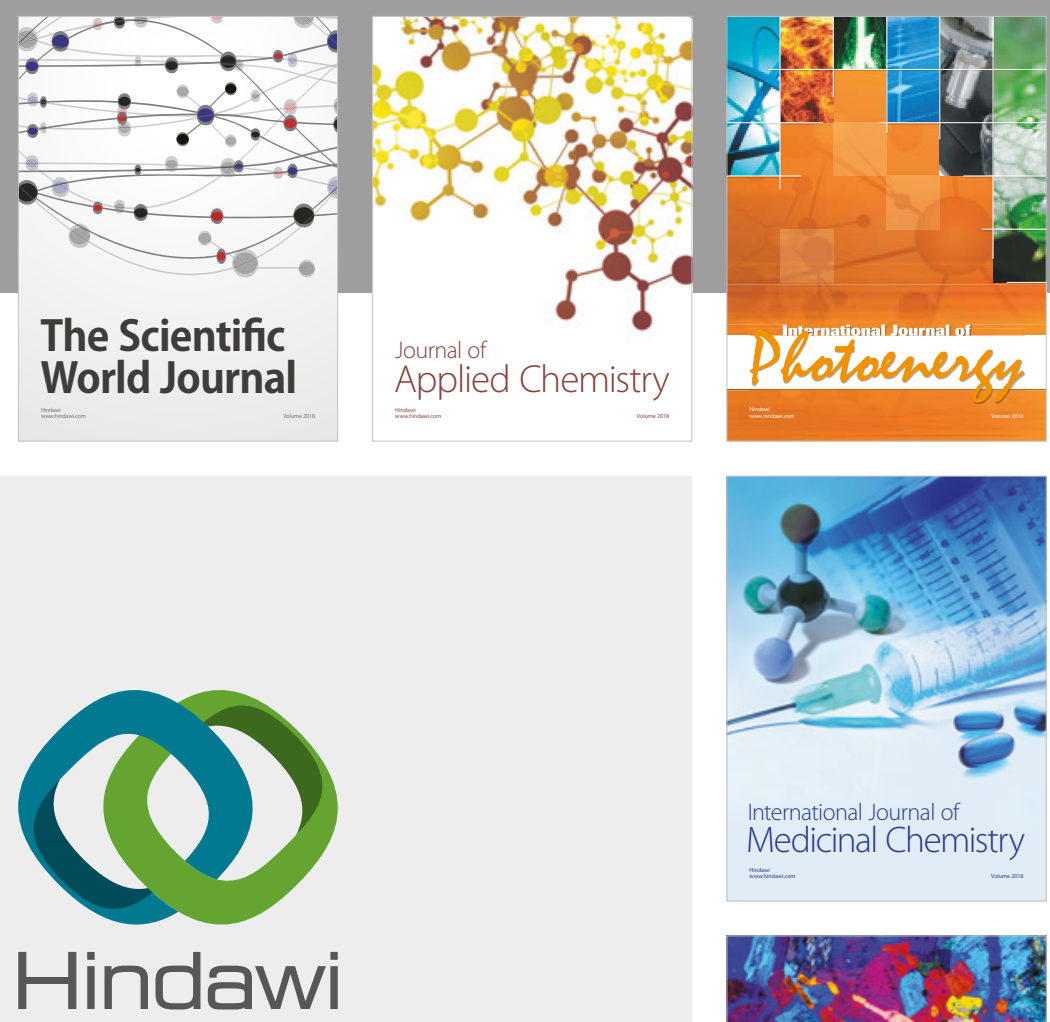

Submit your manuscripts at

www.hindawi.com
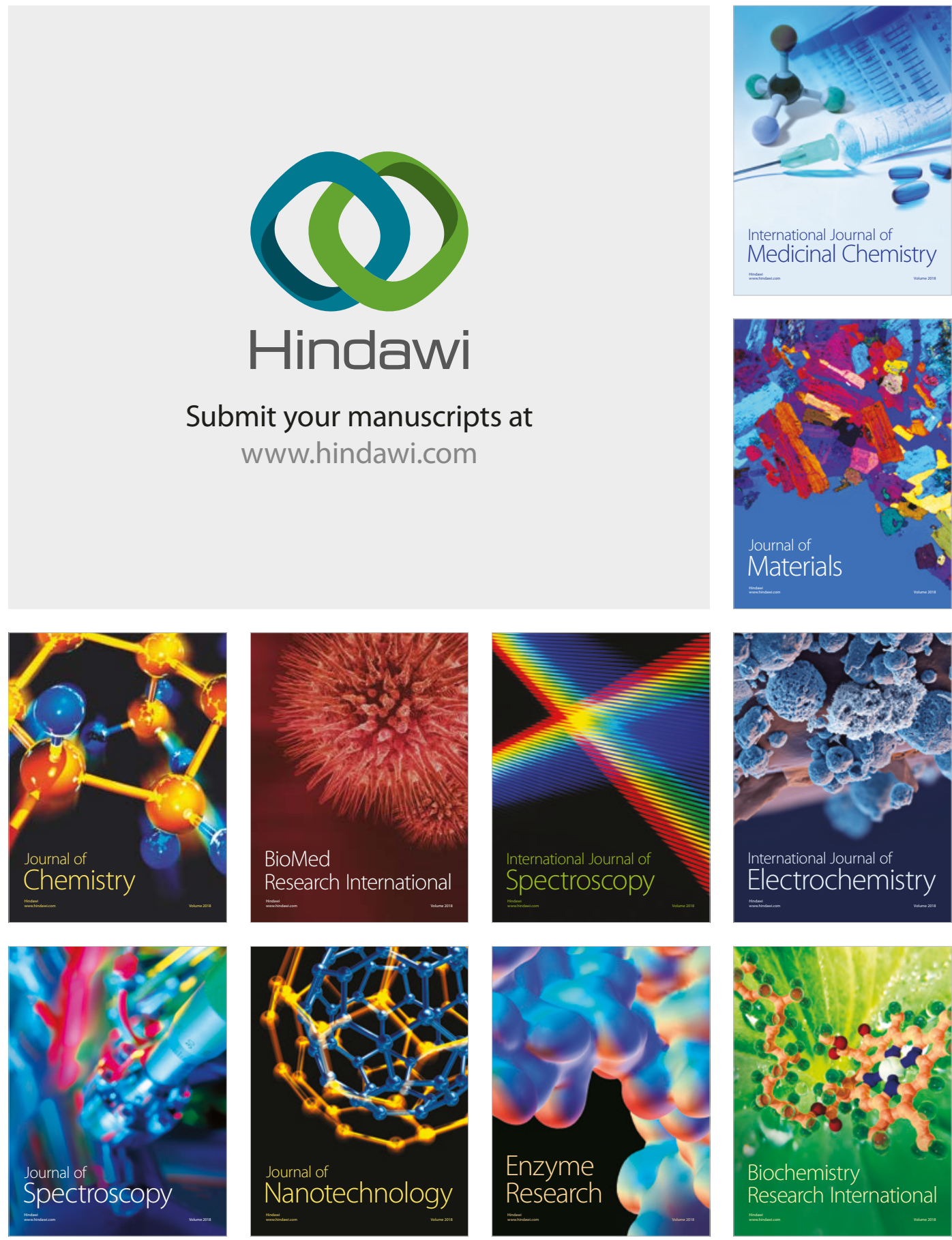
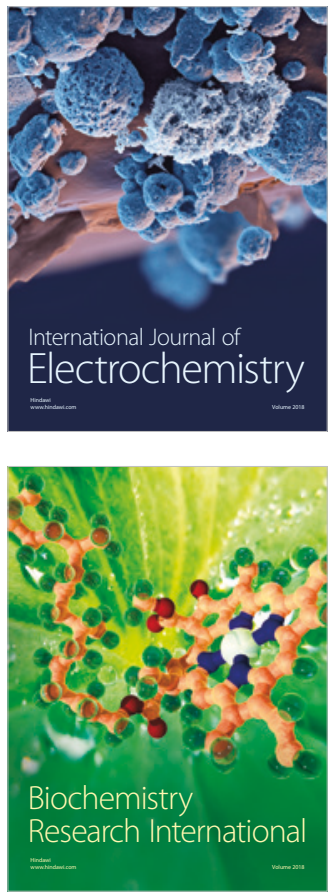